\title{
Transcriptome analysis of IncRNA expression patterns in human congenital lung malformations
}

Weili Yang ${ }^{1}$, Pu Zhao ${ }^{2}$, Yun Liư ${ }^{3}$, Ping Cao ${ }^{1}$, Xiang Ji ${ }^{1}$, Ya Gao ${ }^{1}$, Peng Li ${ }^{* *}$ and Jiwen Cheng ${ }^{{ }^{*}}$

\begin{abstract}
Objectives: To explore the long non-coding RNA (IncRNA) expression pattern of congenital lung malformations on a genome-wide scale and investigate their potential biological function in four subtypes of congenital lung malformations.

Methods: We obtained both lesions and normal lung control tissues from the patients diagnosed with CPAM-I, CPAM-II, ILS, and ILS-CPAM, and underwent lobectomy (i.e., surgical removal of the whole lobe which contains the localized lesion as well as normal lung tissue). Then, we performed IncRNA transcriptome profiling in these tissues by RNA sequencing (RNA-seq). A comprehensive bioinformatics analysis was conducted to characterize the expression profiles and relevant biological functions and for multiple comparisons of IncRNA expression in the different subtypes of congenital lung malformation tissues. Furthermore, the IncRNA-mRNA co-expression network was constructed, and dysregulated mRNAs were functionally analyzed. Finally, gene set enrichment analysis (GSEA) was used to predict the potential molecular mechanism of the identified IncRNAs.
\end{abstract}

Results: A total of 5921 IncRNA transcripts were identified between congenital lung malformations tissues and normal lung control tissues. Compared with normal lung control, 481 of these expressed IncRNAs were upregulated and 142 were downregulated in CPAM-I, 91 were upregulated and 14 were downregulated in CPAM-II, 39 were upregulated and 38 were downregulated in ILS, and 201 were upregulated and 38 were downregulated in ILS-CPAM. Unsupervised clustering and principal component analysis of the expressed IncRNAs visualized the differences between normal lung control and different subtypes of congenital lung malformations samples. We also confirmed significant differences in the composition of differentially expressed genes (DEGs) and the differentially expressed IncRNAs (DE IncRNAs) between CPAM-l and other subtypes of congenital lung malformations, as well as in normal lung control tissues, and observed enrichment of DEGs in the regulation of the immune system, cell projection organization, and inflammatory pathways. Finally, we identified the IncRNA FLJ26850 might be related to congenital lung malformations via ZNF473.

Conclusions: Significant differences in IncRNAs expression patterns were observed between different subtypes of congenital lung malformations and normal control. The IncRNA FLJ26850 might be related to congenital lung malformations via ZNF473.

\footnotetext{
*Correspondence: sienafiat@mail.xjtu.edu.cn; chengjiwen68@163.com

${ }^{1}$ Department of Pediatric Surgery, the Second Affiliated Hospital of Xi'an

Jiaotong University, No. 157, Xiwu Road, Xi'an 710004, Shaanxi, China

Full list of author information is available at the end of the article
} original author(s) and the source, provide a link to the Creative Commons licence, and indicate if changes were made. The images or other third party material in this article are included in the article's Creative Commons licence, unless indicated otherwise in a credit line to the material. If material is not included in the article's Creative Commons licence and your intended use is not permitted by statutory regulation or exceeds the permitted use, you will need to obtain permission directly from the copyright holder. To view a copy of this licence, visit http://creativecommons.org/licenses/by/4.0/. The Creative Commons Public Domain Dedication waiver (http://creativeco mmons.org/publicdomain/zero/1.0/) applies to the data made available in this article, unless otherwise stated in a credit line to the data. 
Keywords: Congenital lung malformations, Transcriptome analysis, IncRNA expression pattern, Co-expression network, Cis regulatory

\section{Background}

Congenital lung malformations include a wide spectrum of developmental abnormalities involving large airways, pulmonary parenchyma, and blood vessels; congenital pulmonary airway malformations (CPAM, previously known as congenital cystic adenomatoid malformations [CCAM]), pulmonary sequestration (further categorized into intralobar sequestrations [ILS] and extralobar sequestrations [ELS]), congenital lobar emphysema (CLE), and bronchogenic cysts are most commonly seen in clinics [1-3]. Typically, lesions occur sporadically without any family inheritance and are only occasionally accompanied by other deformities [4, 5]. Due to their rarity, few reliable data sources can be found on their overall prevalence. But the estimated prevalence of CPAM is 1 per 11,000 to 35,000 live births, comparable to congenital diaphragmatic hernia [4]. Given the increased risk of recurrent pneumonia and theoretical concern for malignant degeneration in later life, pediatric surgeons favor surgical removal of the affected lung in symptomatic infants [6].

Clinically, those lesions are simply grouped by cyst size (microcystic versus macrocystic) and origin of vascular supply (i.e., presence of an anomalous systemic arterial vessel [ILS or ELS], and pulmonary vs. systemic venous drainage) regardless of their heterogeneous and complex nature $[3,7]$. CPAM is usually regarded as the most common type of fetal lung lesion, which was first classified into three subtypes in 1977, and later in 2002, it was reclassified into five subtypes by Stocker based on histology and the presumed site of origin during lung development, with CPAM-I speculatively originating from the tracheobronchial tree, through CPAM-VI, the latter thought to arise from distal alveolar regions [8-11]. Considering the presence of hybrid lesions (such as CPAM with an anomalous systemic arterial vessel, known as ILS combined with CPAM), it is a long-held suggestion that congenital lung abnormalities are part of a continuum of developmental abnormalities instead of separate entities, but the exact etiology and molecular pathogenesis of congenital lung lesions remain unknown $[3,12]$.

Found in every branch of life, long non-coding RNAs (lncRNAs) compose a category of long RNA transcripts with no protein-coding potentiality, enriched in the nucleus or cytoplasm [13]. According to the relationship with coding RNA transcripts, there are five groups of lncRNAs, including sense, anti-sense, intronic, intergenic, and divergent [14]. They can function as signals, sponges/decoys, scaffolds, and guides and play essential roles in various cellular processes, such as dosage compensation, imprinting, transcription/translation, alternative splicing, nuclear-cytoplasmic trafficking, and subcellular localization, etc. [15]. Thus, IncRNAs function critically in both transcriptional and post-transcriptional modifications [16].

To our best knowledge, no studies seem to investigate the regulatory roles of lncRNAs for congenital lung malformations on a genome-wide scale. In the present study, we conducted a combination of transcriptome analyses to explore the molecular pathogenesis of congenital lung malformations (specifically, CPAM, ILS, and hybrid lesions). Then, we determined the DE lncRNAs by using bioinformatics analysis and predicted the differentially expressed mRNAs. Subsequently, the interaction network among lncRNAs and mRNAs was constructed in patients with congenital lung malformations. This study may promote our understanding of the role of lncRNAs and associated pathways in human congenital lung malformations.

\section{Results}

Genome-wide profiling of the IncRNA expression associated with congenital lung malformations

In the present study, we used a whole-genome RNA-seq dataset recently generated from 15 samples containing three CPAM-I, three CPAM-II, three ILS, three ILSCPAM lung tissue samples, and three normal lung control samples from patients undergoing surgical resection of congenital lung malformations. A subset of lncRNAs showed remarkable expression changes in different subtypes of congenital lung malformations compared to the normal lung control tissues, as shown in the Venn diagram, the bar plot, and the clustered heatmaps (Fig. 1a-c).

Moreover, we observed that both the known lncRNAs and the novel lncRNAs showed remarkable expression changes in the different subtypes of congenital lung malformations tissues and normal lung control tissues using the Venn diagram (Fig. S1A and B). We also found gene length distribution and exon count of the known lncRNA, the novel lncRNA, and the protein-coding RNA were significantly different in the different subtypes of congenital lung malformations tissues and normal lung control tissues (Fig. S1C and D). Next, we observed the principal component of DEGs and DE lncRNAs in congenital lung malformations tissues and normal lung control tissues and found that significant differences in the 
A

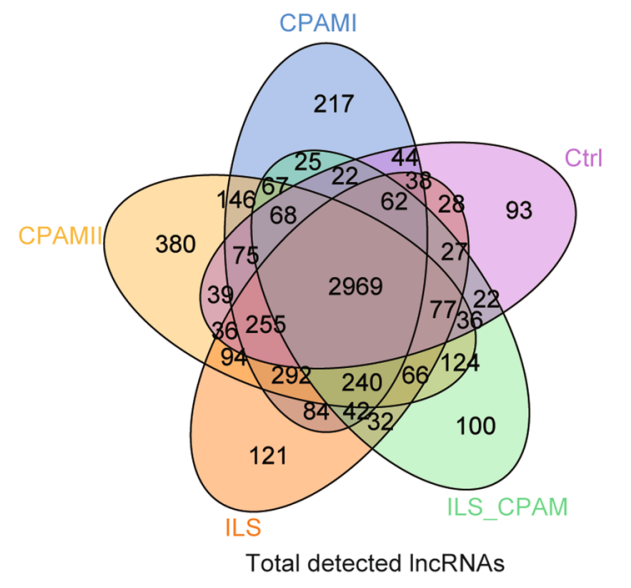

D

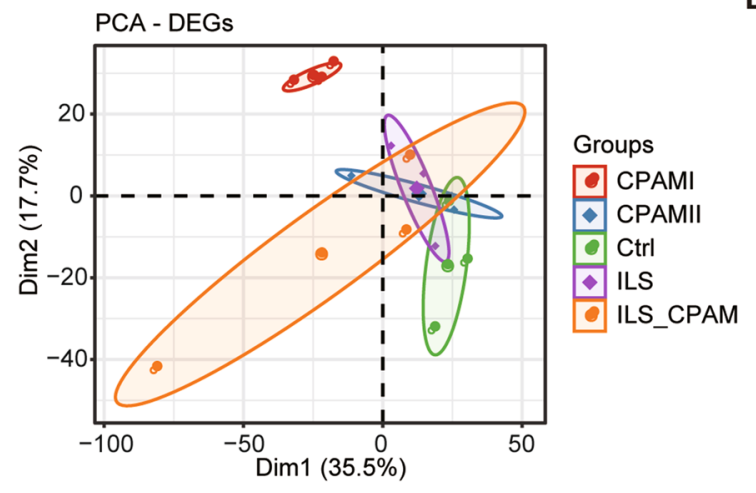

B

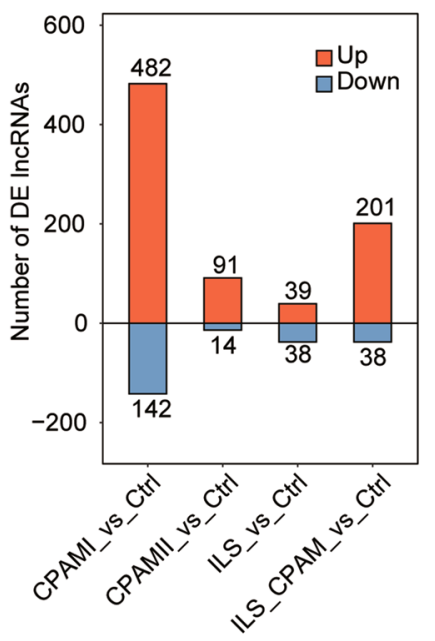

C

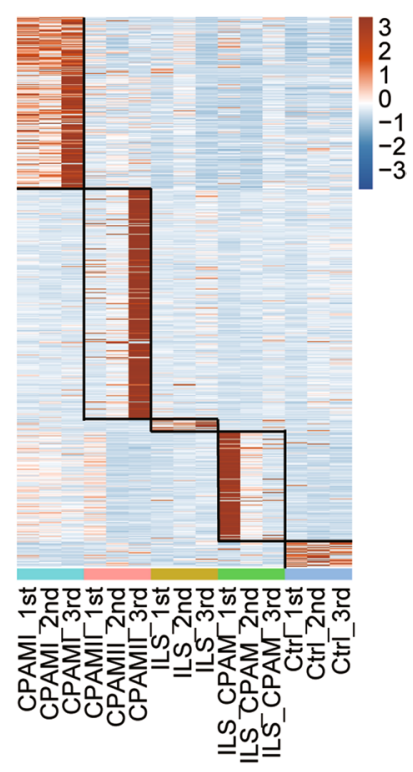

E

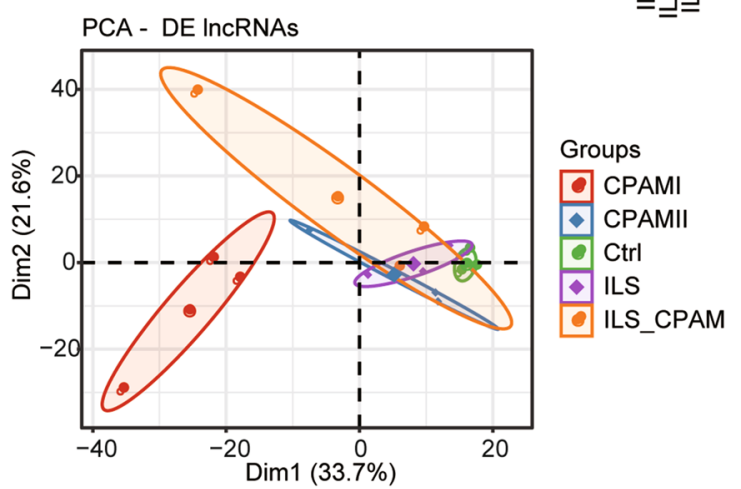

Fig. 1 A comprehensive catalog of IncRNA expression pattern in congenital lung malformation tissues. a. The Venn diagram of detected IncRNA data from 15 samples (three CPAM-I, three CPAM-II, three ILS, three ILS hybrid CPAM, and three normal lung control samples obtained from patients undergoing lobectomy). b. The bar plot illustrates the number of differentially expressed genes between subtypes of congenital lung malformation with paired control lung tissue. c. Heatmap of all congenital lung malformation-specific IncRNAs. The color key from blue to red indicates the z-score color range. d. Principal component analysis (PCA) of 15 samples based on normalized differentiation gene expression level. All CPAM-I samples grouped together were used for the subsequent analyses. e. Principal component analysis (PCA) of 15 samples based on normalized DE IncRNA expression level

composition of DEGs and lncRNAs between CPAM-I and other subtypes of congenital lung malformation, as well as in normal control (Fig. 1d and e, Fig. S1E and F). The above results indicated that significant differences in IncRNAs expression patterns existed among different subtypes of congenital lung malformations and normal control.

\section{Analysis of the lesion type specific-IncRNA and their co-expressed mRNAs in CPAM-I and other lesion tissues} Given the distinction between CPAM-I and other subtypes of congenital lung malformations, we analyzed the DE lncRNAs and their co-expressed mRNAs in CPAM-I tissues. The scatter plot shows the DE
lncRNAs of CPAM-I compared with normal lung control samples and the number of their co-expressed DEGs, and up and down-regulated lncRNAs (Fig. 2a, Fig. S2A-C). The GO analysis and KEGG pathway analysis of the co-expression mRNAs in CPAM-I and normal control tissues indicated enrichment in the immune response, cell projection organization, and inflammatory response; additionally, the co-expression mRNAs in CPAM-II and normal control tissues indicated enrichment in the immune response; the coexpression mRNAs in ILS and normal control tissues indicated enrichment in the cellular defense response, and co-expression mRNAs in ILS-CPAM and normal control tissues indicated enrichment in the immune 


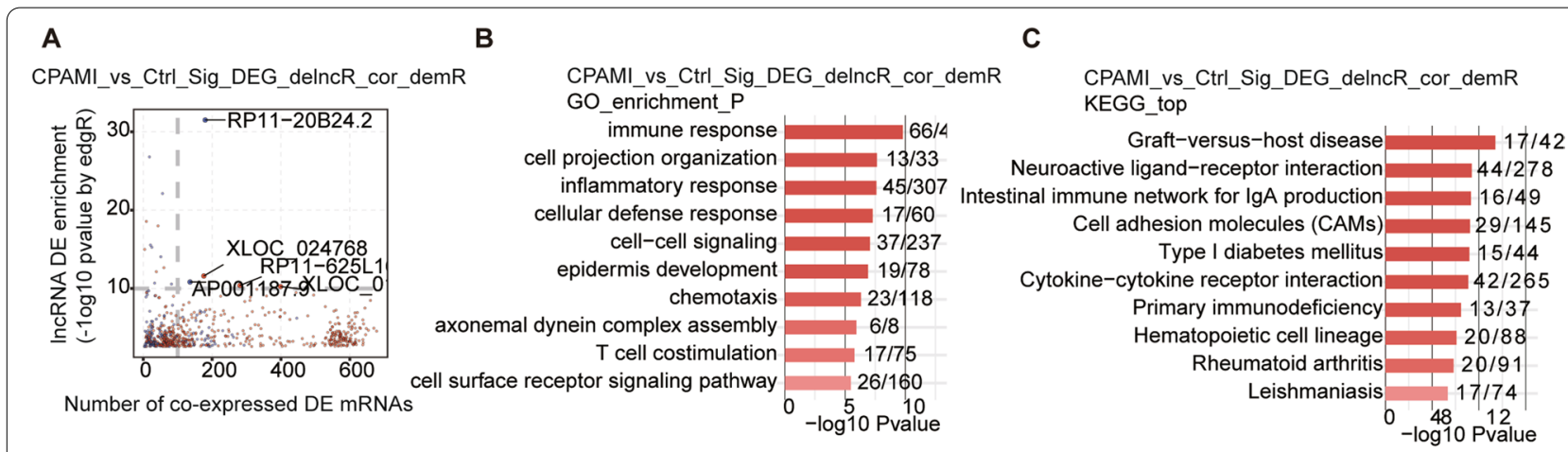

D

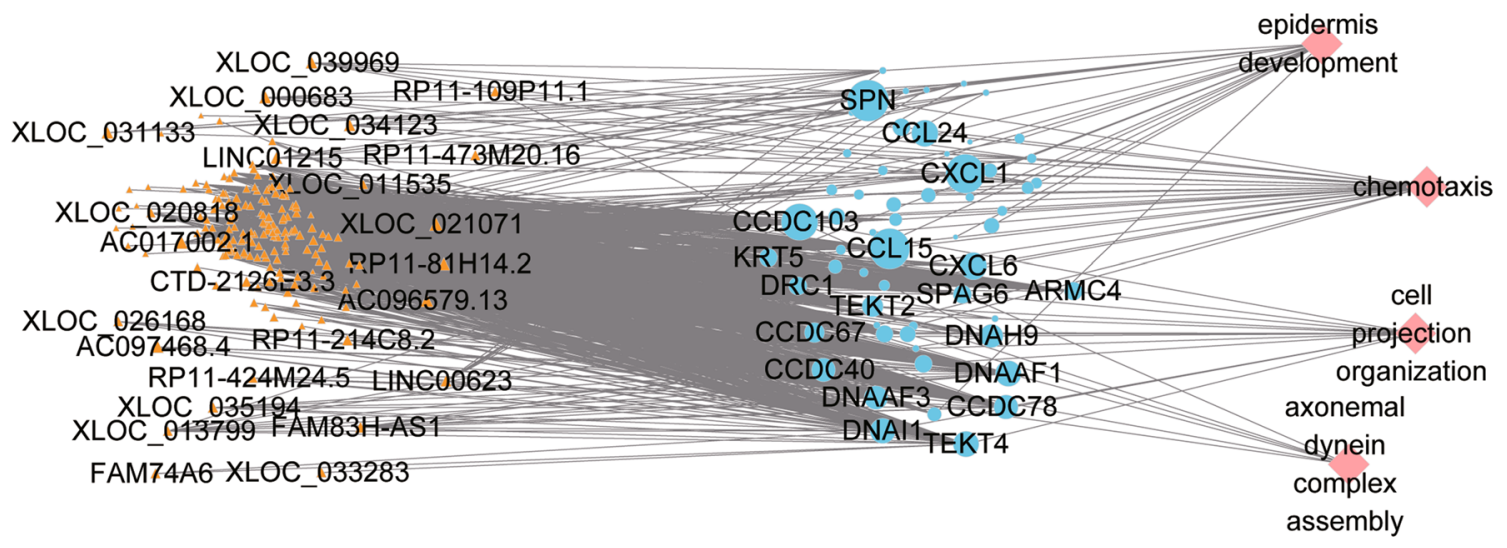

E
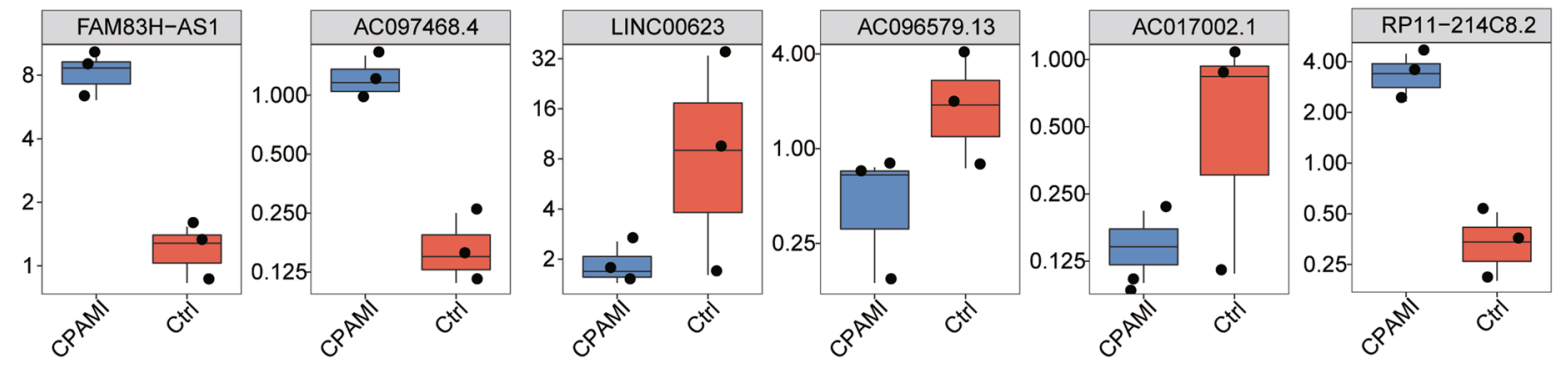

Fig. 2 Co-expression network illustration of IncRNA-mRNA and functional analysis of target mRNAs in CPAM-I lung tissues. a. Scatter plot shows DE-InCRNAs of CPAM-I compared with control samples and the number of co-expressed DE mRNAs. Up and down-regulated IncRNAs are respectively labeled in red and blue points. Cutoffs of $p$-value $<0.01$ and Pearson coefficient $>0.6$ were applied to identify the co-expression pairs. b. Top 10 most enriched GO terms (biological process) by DE mRNAs co-expressed with DE-IncRNAs of CPAM-I lung tissues compared with control samples. c. The top 10 enriched KEGG pathways by DE mRNAs co-expressed with DE-IncRNAs of CPAM-I lung tissues compared with control samples. $\mathbf{d}$. The co-expression network between the IncRNAs detected in the CPAM-I lung tissues and the co-expressed mRNAs involved in the four GO terms. IncRNA are plotted with yellow circles, co-expression mRNAs are plotted with light blue circles, mRNA-enriched GO terms are plotted with red circles. e. The expression level of the six IncRNAs in CPAM-I lung tissues

response, cell projection organization, and cellular defense response (Fig. 2b and c, Fig. S2D-I). Moreover, we constructed the co-expression network between the lncRNAs detected in the CPAM-I lung tissues and coexpressed mRNAs and found four GO terms involved in this co-expression network (Fig. 2d). Subsequently, we detected the expression level of the top six most
DE lncRNAs and found that lncRNA FAM83H-AS1, lncRNA AC097468.4, and lncRNA RP11-214C8.2 were significantly upregulated, and IncRNA LINC00623, lncRNA AC096579.13, and lncRNA AC017002.1 were significantly downregulated (Fig. 2e). 


\section{WGCNA analysis of the IncRNA and mRNA co-expression in CPAM-I clinical samples}

To further define the changes of DE lncRNAs in CPAM-I lung tissues, we evaluated the expression patterns of $\mathrm{DE}$ lncRNAs and co-expression mRNAs in CPAM-I lung tissues and normal control tissues by WGCNA analysis and found that CPAM-I samples had eight modules with a decreased expression, five modules with an increased expression in the disease samples with four modules at the location of the dashed line. These results represented the four modules involved in congenital lung malformations (Fig. 3a, Fig. S3A and B). Then, we detected the expression fold change of mRNAs and lncRNAs from the four CPAM-I associated modules and found that the fold changes in the expression levels of the corresponding mRNAs and lncRNAs were consistent (Fig. 3b). Furthermore, we analyzed the top 10 hub genes and/or at least five top hub lncRNAs along with the GO terms enrichment of each of the four modules. The brown module indicates the enrichment in the ovarian steroidogenesis and arachidonic acid metabolism, the green-yellow module indicates the enrichment in the protein digestion and absorption, folate biosynthesis, and cell adhesion molecules (CAMs), the yellow module indicates the enrichment in alcoholism, taste transduction, GABAergic synapse, and morphine addiction, and the pink module indicates the enrichment in the protein digestion and absorption (Fig. 3c-f).

\section{Analysis of cis-acting IncRNA-regulated mRNA network in congenital lung malformations}

To characterize the function of DE lncRNAs in cis-regulation, their adjacent protein-encoding genes, which were placed $10 \mathrm{~kb}$ of lncRNAs downstream, were chosen to conduct co-expression analysis. In total, 151 lncRNAmRNA pairs probably associated with cis-acting regulation were found (Fig. 4a). Cis-acting IncRNA-regulated mRNA indicated enrichment in the positive regulation of transcription from RNA polymerase-II promoter, regulation of transcription, and DNA dependent (Fig. 4b, Fig. S4A). Subsequently, we highlighted two DE lncRNAs FLJ26850 and PSORS1C3, both of which interacted with miRNA processing complexes and were typically upregulated in congenital lung malformations (Fig. 4c and d, Fig. S4B and C). These data showed that the dysregulation of lncRNAs, many of which are congenital malformation lung tissue-enriched, affected the mRNAs through the cis-regulatory target.

\section{Discussion}

The first identification of congenital lung lesions was over a century, but their etiology and pathogenesis only have been partially unveiled [2, 7]. Different classification systems and terminologies have evolved to describe this disorder in clinical practice, yet the differential expression and regulatory mechanisms of IncRNAs in different subtypes of congenital lung malformations are unknown. In this study, we screened the genome-wide transcripts comprehensively to identify regulating lncRNAs in the different subtypes of congenital lung malformations with RNA-seq data. Our analysis suggested that the dysregulated lncRNAs were mostly involved in the following pathways: immune response, cell projection organization, and inflammation. Noticeably, the lncRNA FLJ26850 might be linked to congenital lung malformations via the ZNF473-mediated biological process. Overall, our results implicated the potential regulatory roles of several lncRNAs in congenital lung malformations.

The mammalian genome encodes a large number of lncRNAs, which play key functional roles in biological processes including cell proliferation and differentiation $[17,18]$. In our study, many mRNAs were found regulated by multiple differential lncRNAs, such as HBD, HBG2, FAM196A, AHSP, and COL1A1. With the development of high-throughput technologies, large amounts of microarray and RNA- seq data have been obtained, but few studies have characterized lncRNA expression profile in congenital lung malformations. In our analysis, we characterized the expression profile of lncRNAs in congenital lung malformations by RNA-seq and identified 5921 expressed lncRNAs between different subtypes of congenital lung malformations tissues and normal lung control tissues. From the GO and pathway analyses of lncRNAs co-expressed mRNAs, we found that these significantly DE lncRNAs were associated with the immune response and inflammatory pathways. The results imply that they might play important roles in congenital lung malformations.

Congenital lung lesions usually display relatively mature airway structures of varying morphological features, indicating substantial heterogeneity in histology both within and across lesion types, even within individual

\footnotetext{
(See figure on next page.)

Fig. 3 WGCNA analysis of expression pattern of differentially expressed IncRNAs and mRNAs in CPAM-I lung tissues. a. Signed association of module eigengenes with the diagnosis of CPAM-I. Positive values indicate modules with an increased expression in the disease samples. Negative values indicate modules with a decreased expression in the disease samples. The dashed lines signifies the disease-associated modules. $\mathbf{b}$. The boxplot shows the expression fold change of mRNAs and IncRNAs from the four CPAM-I associated modules. c-f. The module plots display the top 10 hub genes and/or at least five top hub IncRNAs along with the GO terms enrichment of each of the four modules. Brown module (c), Greenyellow module (d), Yellow module (e), Pink module (f). Orange circles indicate IncRNAs and light blue circles indicate mRNAs
} 
A

C

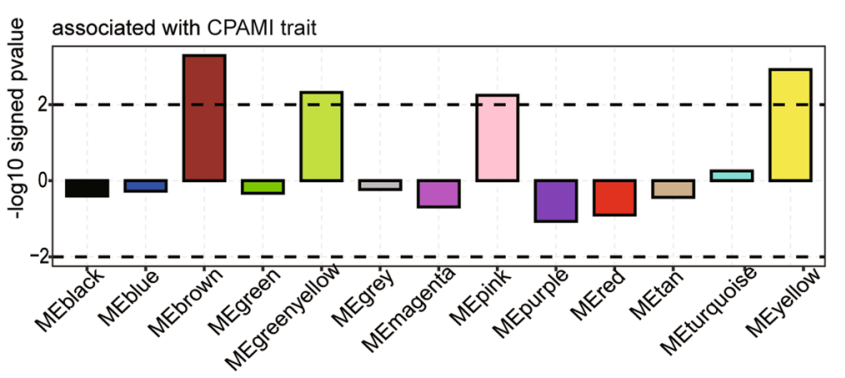

B

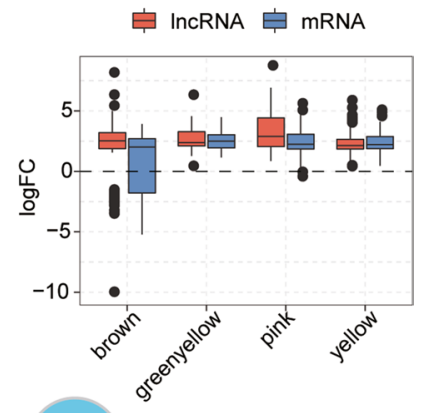

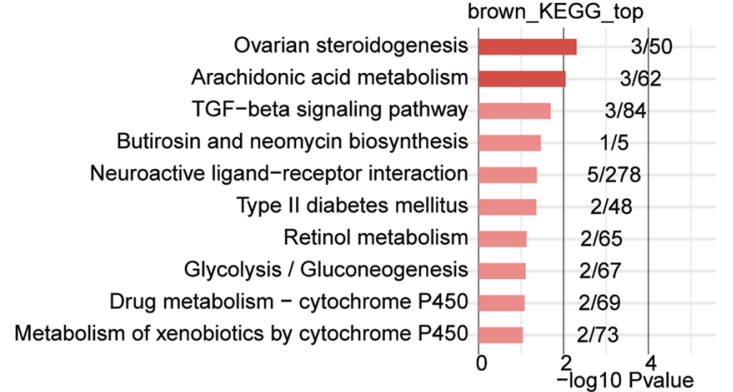

greenyellow_KEGG_top

D

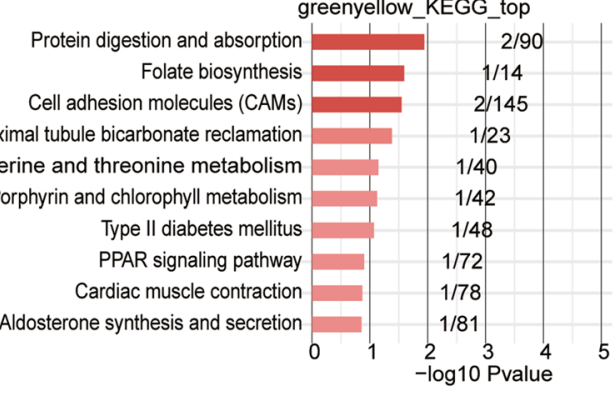

E

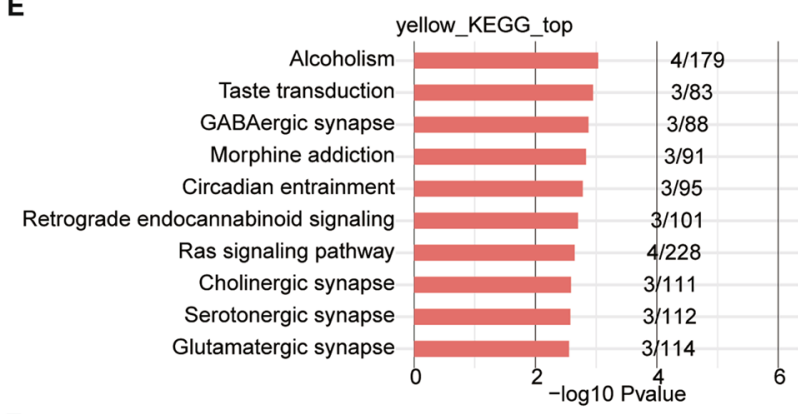

$\mathbf{F}$

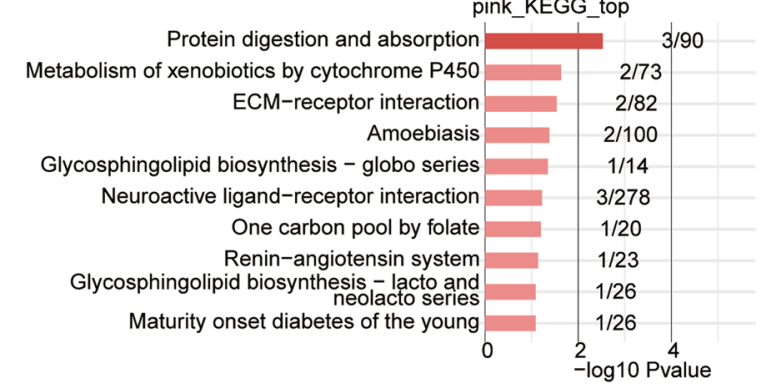

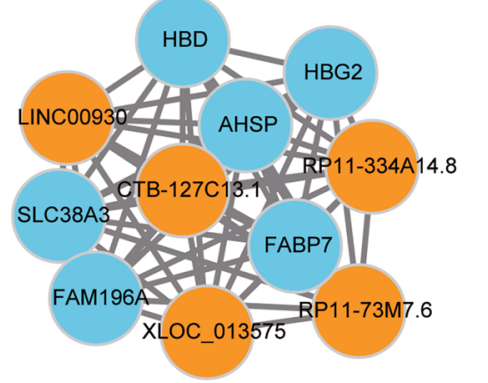
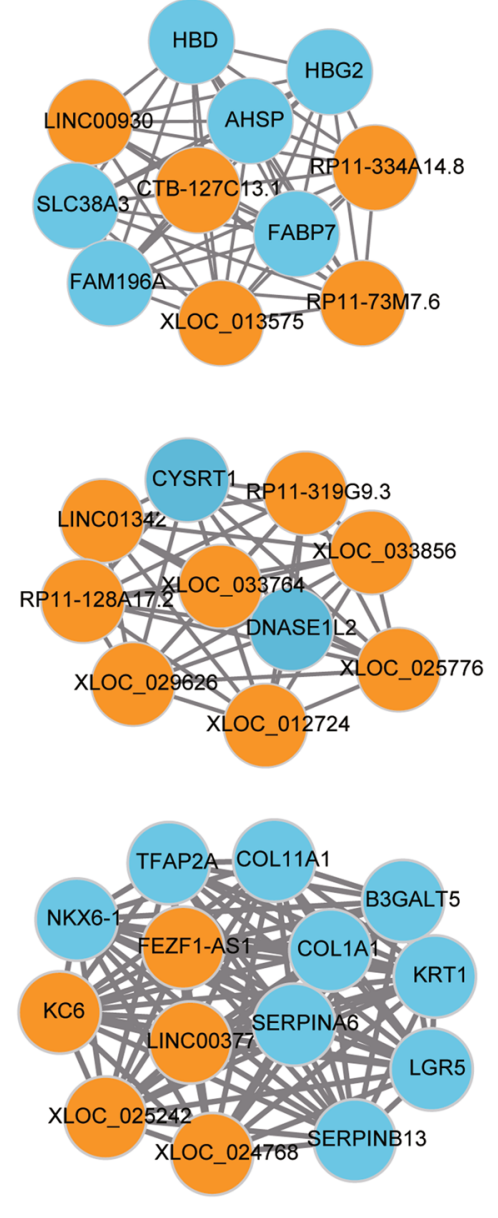

Fig. 3 (See legend on previous page.) 

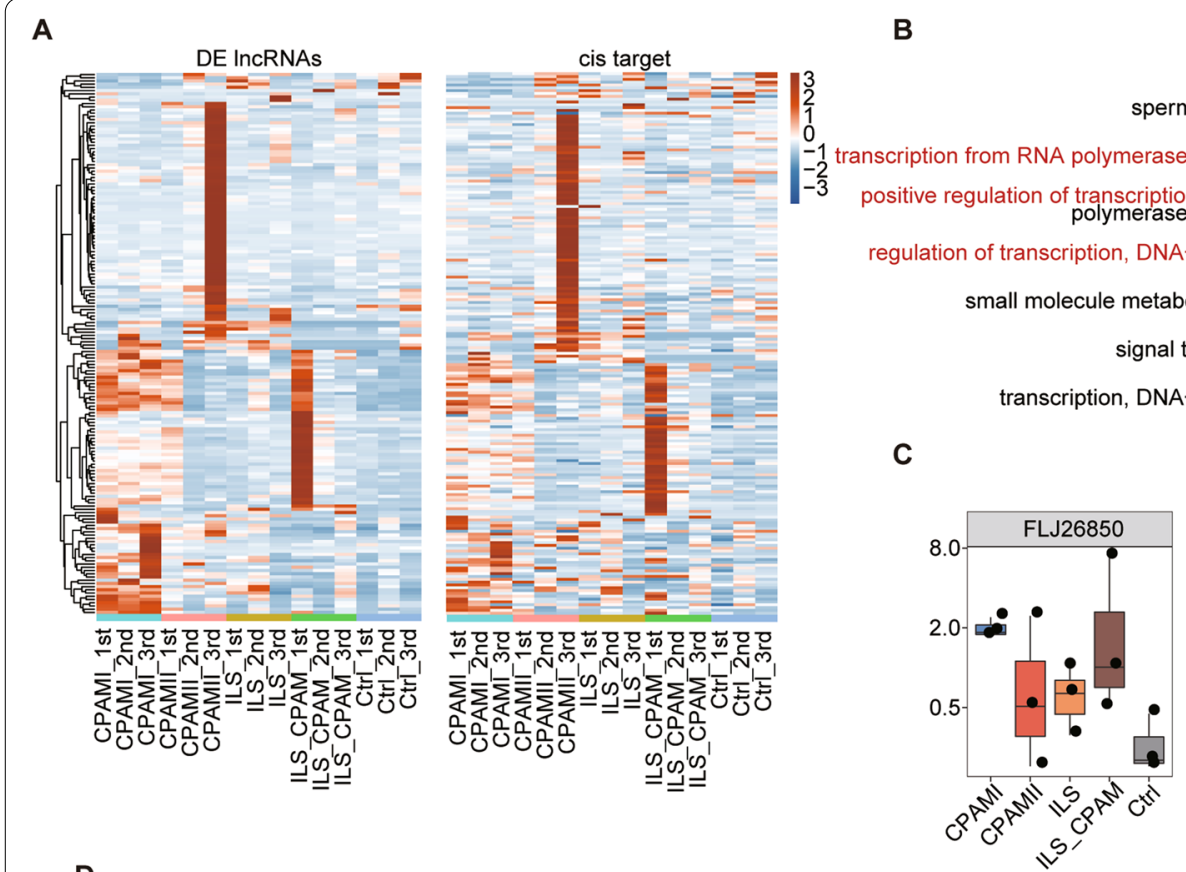

GO BP top10 (IncRNA cis target)

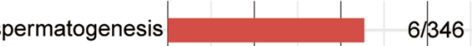

promoter

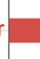

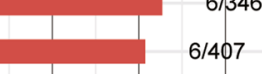

pendent

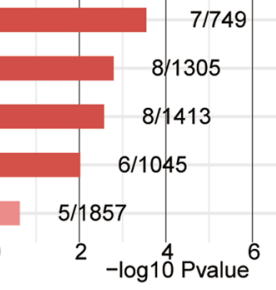

D

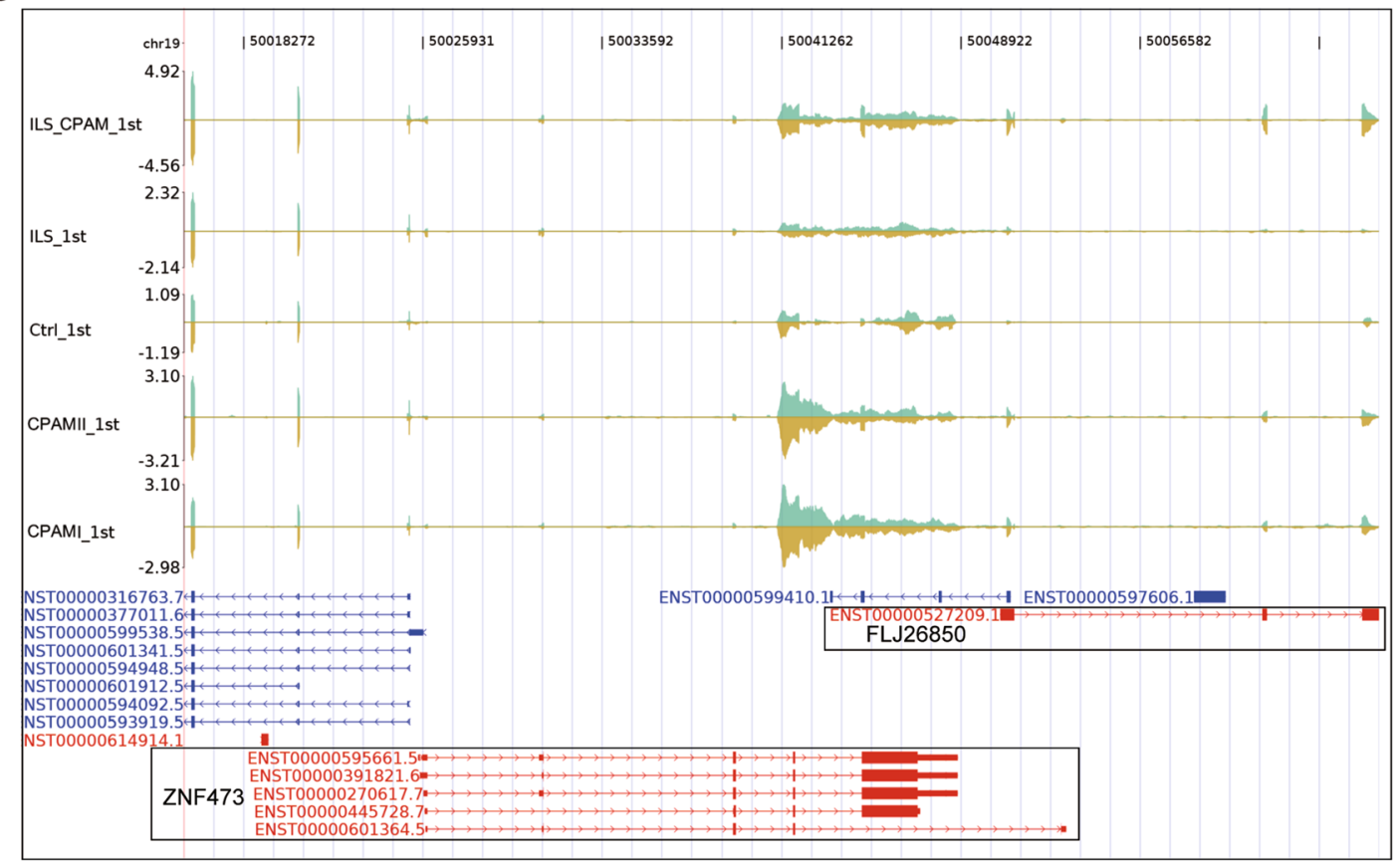

Fig. 4 Cis-regulatory target genes of DE IncRNAs in all congenital lung malformation tissues. a. Heatmap shows the expression pattern of DE IncRNAs and their cis-regulatory genes. b. Top 10 most enriched GO terms of the cis-regulatory genes. c. The expression level of IncRNA FLJ26850 and its cis-regulatory target ZNF473 in all congenital lung malformation tissues. d. Visualization of IncRNA FLJ26850 and its cis-regulatory target ZNF473s

lesions $[19,20]$. Specifically, recent research has further reported unequal histopathologic characteristics and gene expression changes associated with the pathogenesis of these lesions [11, 21-23]. The gene expression dataset generated by our current study contributes to the comprehensive view of the transcriptional changes within various common types of congenital lung lesions. We observed a significant enrichment of known ZNF473 targets among the down-regulated transcripts. Also, a couple of molecular pathways are suspected to involve in 
the pathogenesis by the GSEA data, which may provide certain directions for future studies.

\section{Conclusion}

In this study, we ascertained the differential expression of lncRNAs in congenital lung malformations tissues compared with normal lung control tissues using RNA-seq. And the results of the GO and pathway analyses showed that certain lncRNAs might play key roles in the development of congenital lung malformations. Moreover, in these lncRNAs, we found the most dysregulated lncRNA FLJ26850 in congenital lung malformations. It could be used as a prognostic biomarker and has the potential to be a diagnostic biomarker and a new therapeutic target for congenital lung malformations. Although our findings are preliminary, while our study promotes understanding of the pathogenesis of congenital lung malformations and provides potential biomarkers for diagnosis and treatment of congenital lung malformations.

\section{Materials and methods}

All methods were carried out in accordance with relevant guidelines and regulations.

\section{Patients and specimens}

We recruited 1-year-old infants with a confirmed diagnosis of CPAM-I $(N=3$, mean \pm SE: $2.89 \pm 0.67$ months, including one male), CPAM-II $(N=3$, mean \pm SE: $3.15 \pm 0.84$ months, including one male), ILS $(N=3$, mean \pm SE: $6.37 \pm 1.96$ months, including two males) and ILS- CPAM $(N=3$, mean \pm SE: $4.03 \pm 0.97$ months, including two males) into our study. Normal lung control samples $(N=3$, mean \pm SE:4.81 \pm 0.87 months, includes 2 males) were obtained from patients undergoing resection of congenital lung malformations at the Second Affiliated Hospital of Xi'an Jiaotong University from May 2017 to August 2018. Specimens from each patient were then divided into two portions. The first portion was fixed in buffered formalin for histopathologic examination, and the second one was immediately snap-frozen in liquid nitrogen for RNA-seq analysis. The study protocol was approved by the Institutional Ethics Board of Xi'an Jiaotong University and written informed consent was obtained from the guardians of all subjects.

\section{RNA preparation and sequencing}

Total RNA including the lncRNA (miRNA) fraction was isolated with High Pure miRNA isolation kit (Cat no: 05080576001, Roche, Penzberg, Germany) using the onecolumn protocol according to the manufacturer's recommendation. RNA quantity was measured on a Qubit fluorometer with the Qubit RNA Assay Kit (Life Technologies, Eugene, OR, USA) and also on the NanoDrop-1000 instrument (Thermo Fisher Scientific Inc., Waltham, USA) to determine the purity values (OD260/280, OD260/230). RNA quality analysis was performed on an Agilent Bioanalyzer microcapillary electrophoresis system with the RNA 6000 Pico Kit (Agilent, Santa Clara, CA, USA).

\section{RNA-Seq raw data clean and alignment}

We first discarded raw reads with more than 2-N bases. Then, we used FASTX-Toolkit (Version 0.0.13) to trim adaptors and low-quality bases from raw sequencing reads. We also dropped reads shorter than $16 \mathrm{nt}$. Next, we aligned those clean reads to the GRCH38, version 23 (Ensembl 81) (https://www.ebi.ac.uk/about/news/servi ce-news/ensembl-version-81-release) using TopHat v2 with four mismatches. The RNAseq mapping statistics can be found in Supplementary Material Table 1). Further, we used uniquely mapped reads to count the number of gene reads and calculate FPKM (fragments per kilobase of transcript per million fragments mapped). The cleaned data for each sample were saved in FASTQ format. FastQC (http://www.bioinformatics.babraham. ac.uk/projects/fastqc/) was used to assess the quality of the raw reads.

\section{Reverse transcription-quantitative polymerase chain reaction (RT-qPCR) [24]}

After RNA isolation, M-MLV reverse transcriptase (Invitrogen, USA) was used for synthesizing cDNA according to the manufacturer's instructions. Subsequently, we performed RT-qPCR by SYBR Green assays in a total reaction volume of $20 \mu \mathrm{L}$, including $0.8 \mu \mathrm{L}$ of PCR Forward Primer $(10 \mu \mathrm{M}), 0.8 \mu \mathrm{L}$ of PCR Reverse Primer $(10 \mu \mathrm{M})$, $2 \mu \mathrm{L}$ of cDNA, $5 \mu \mathrm{L}$ of $2 \times$ Master Mix and $12.8 \mu \mathrm{L}$ of double distilled water. The RT-qPCR cycling conditions consisted of $95^{\circ} \mathrm{C}$ for $3 \mathrm{~min}$; then 35 cycle amplification for $35 \mathrm{~s}$ at $95^{\circ} \mathrm{C}, 45 \mathrm{~s}$ at $58^{\circ} \mathrm{C}, 30 \mathrm{~s}$ at $72^{\circ} \mathrm{C}$; followed by $10 \mathrm{~min}$ at $72^{\circ} \mathrm{C}$. $\beta$-actin was used as a housekeeping gene for normalization, and the relative change in gene expression was analyzed by the $2^{-\Delta \Delta C T}$ method.

\section{Principal component analysis, hierarchical clustering and visualization}

Firstly, we used the TCGAanalyze_Normalization function for quantile-normalization of raw counts both within and across samples; then, to control heteroscedasticity, the counts were transformed using the varianceStabilizingTransformation function in DESeq2 (chosen over log transformation to minimize variance at low count values) [25]. We used the prcomp function in $\mathrm{R}$ to conduct principal component analysis (PCA)(https://cloud.rproject.org/package=factoextra) [26]. To get hierarchical clusters, the Euclidean distance was used to measure 
the dissimilarity following the Ward2 criterion [27]. To determine how much variance may be explained by the variables, we first used the $\mathrm{lm}$ function to construct multiple linear regression models for response variables "PC1" and "Cluster", respectively. Next, the relaimpo package was chosen to determine the relative contribution of explanatory variables to the variance [28]. The PCA results were plotted using the plotPCA function in DESeq2 for visualization [25]. All heatmaps were constructed with the ComplexHeatmap R package [29].

\section{Correlation and co-expression network analysis}

After calculating the Pearson correlation coefficient between lncRNAs and mRNAs based on differentially expressed mRNAs and lncRNAs, those lncRNAs and mRNAs whose expression levels showed meaningful correlations $(P$-value $<0.05)$ were subjected to the co-expression analysis. For each gene pair (including lncRNAs and protein-coding genes), we used the WGCNA package in $\mathrm{R}$ to calculate the Pearson correlation coefficients and corresponding $P$-values [30]. All $P$-values were adjusted via Bonferroni correction using the multtest $\mathrm{R}$ package to account for multiple testing [31]. Gene/lncRNA clusters with high interconnections were detected by Markov clustering (MCL) [32]. Bonferroni-adjusted $P$-values (cutoff: 0.01) were used as edge weights for MCL.

\section{Gene Ontology (GO) and pathway enrichment analysis}

We performed Gene Ontology analysis (GO, http:// geneontology.org/) for the meaningful annotations of genes and gene products, which has covered various domains of biological processes, cellular components, and molecular functions. The - $\log 10$ ( $P$-value) denotes enrichment score, which represents the significance of GO term enrichment among differentially expressed genes. We also conducted Kyoto Encyclopedia of Genes and Genomes pathway analysis (KEGG, http://www. kegg.jp/) [33] to harvest pathway clusters along with our knowledge on the molecular interactions and reaction networks in differentially regulated gene profiling. Furthermore, the - $\log 10$ ( $P$-value) denotes enrichment score showing the significance of the pathway correlations.

\section{Cis-regulation prediction [34]}

A cis-regulator exerts its function on a neighboring gene located at the same chromosome [35]. After we identified the significantly changed lncRNAs whose expression levels were correlated with that of mRNAs, they were subjected to cis prediction. The genomic localization of the paired lncRNAs and mRNAs was identified. If the nearby gene is less than $10 \mathrm{~kb}$ downstream away from the IncRNA, it is assumed to be the putative target regulated by that lncRNA in a cis manner. With this criterion, we enriched those mRNAs co-expressed with lncRNAs significantly overlapped with the target genes to construct the lncRNAs-mRNAs network.

\section{Abbreviations}

IncRNA: Long non-coding RNA; DEGs: Differentially expressed genes; DE IncRNAs: Differentially expressed IncRNAs; CPAM: Congenital pulmonary airway malformations; ILS: Intralobar sequestrations; ELS: Extralobar sequestrations; CLE: Congenital lobar emphysema; RT-qPCR: Reverse transcription-quantitative polymerase chain reaction; PCA: Principal component analysis; GO: Gene ontology; RNA-seq: RNA sequencing; CAMs: Cell adhesion molecules.

\section{Supplementary Information}

The online version contains supplementary material available at https://doi. org/10.1186/s12864-021-08204-x.

Additional file 1: Supplementary Material Table 1. RNA-seq Mapping Statistics.

Additional file 2: Figure S1. Characteristics of IncRNAs detected in all congenital lung malformation tissues. A. Venn diagram of detected known IncRNA in human congenital lung malformation tissues.s At least two samples with RPKM $>=0.2$ were considered to be detected in the group. B. Venn diagram of detected novel IncRNA (right) in human congenital lung malformation tissues. At least two samples with RPKM $>=0.2$ were considered to be detected in the group. C. Distribution of gene length distribution of known IncRNA, novel IncRNA, and protein coding RNA. The length density distribution was generated by density function in R. D. Distribution of exon count of known IncRNA, novel IncRNA, and protein-coding RNA. E-F. Principal component analysis (PCA) of all samples based on all normalized mRNAs (E) and IncRNAs (F) expression levels. The samples were grouped by disease state and the ellipse for each group is the confidence ellipse.

Additional file 3: Figure S2. Co-expression network illustration between DEIncRNAs and DEmRNAs. A-C. Scatter plots show DE IncRNAs by CCAM II (A), ILS_CCAM (B), ILS (C) compared with control samples and their number of co-expressed DE mRNAs. Red points denote up-regulated IncRNAs involved in co-expression pairs, and blue points denote down-regulated IncRNAs. Cutoffs of $P$-value $<0.05$ and Pearson coefficient $>0.6$ were applied to identify the co-expression pairs. D. The top 10 most enriched GO terms (molecular process) by DE mRNAs co-expressed with DE-IncRNAs of CCAM II lung tissues compared with control samples. E. The top 10 enriched KEGG pathways by DE mRNAs co-expressed with DE-IncRNAs of CCAM II lung tissues compared with control samples. F. The top 10 most enriched GO terms (molecular process) by DE mRNAs co-expressed with DE-IncRNAs of ILS lung tissues compared with control samples. G. The top 10 enriched KEGG pathways by DE mRNAs co-expressed with DE-IncRNAs of ILS lung tissues compared with control samples. H. The top 10 most enriched GO terms (molecular process) by DE mRNAs co-expressed with DE-IncRNAs of ILS_CCAM lung tissues compared with control samples. I The top 10 enriched KEGG pathways by DE mRNAs co-expressed with DEInCRNAs of ILS_CCAM lung tissues compared with control samples.

Additional file 4: Figure S3. WGCNA analysis of all expressed IncRNAs and mRNAs. A. Hierarchical cluster dendrogram of all differentially expressed IncRNAs modules. Modules corresponding to branches are labeled with colors indicated by the color bands underneath the tree. B. Module-trait associations as computed by an LME model with all factors on the $\mathrm{x}$-axis used as covariates. All Pearson's correlation values and $p$ values are displayed.

Additional file 5: Figure S4. Cis regulatory genes of DE IncRNAs. A. Top 10 most enriched Reactome pathways of cis-regulatory genes. B. Expression level of IncRNA PSORS1C3 and its cis-regulatory target POU5F1. C. Visualization of IncRNA PSORS1C3 and its cis-regulatory target POU5F1. 


\section{Acknowledgments \\ Not applicable.}

\section{Authors' contributions}

WY carried out the DEGs identification, participated in the statistical analysis, and drafted the manuscript. PZ performed the statistical analysis, participated in DE IncRNAs identification, prepared the figures and tables and drafted the work and revised it critically for important content. YL carried out the conserved domains and gene cluster analysis and helped to prepare the figures and Tables. XJ and PC collectd specimens. JC, PL and YG conceived the study and participated in its design and coordination. All authors read and approved the final manuscript.

\section{Funding}

This work was supported in part by the National Natural Science Foundation of China (Grant no. 81800450), the Key Research and Development plan of Shaanxi Province (Grant no. 2018SF-22), and the Natural Science Foundation of Shaanxi Province, China (Grant no. 2021 JM-298).

\section{Availability of data and materials}

All the raw data has been deposited to the GEO (Gene Expression Omnibus), with the accession number of GSE179404 (https://www.ncbi.nlm.nih.gov/ geo/query/acc.cgi?acc=GSE179404).

\section{Declarations}

\section{Ethics approval and consent to participate}

The study protocol was approved by the Institutional Ethics Board of Xi'an Jiaotong University and a written informed consent was obtained from the guardians of all subjects. All methods were carried out in accordance with relevant guidelines and regulations.

\section{Consent for publication}

Not applicable.

\section{Competing interests}

The authors declare that they have no competing interests.

\section{Author details}

'Department of Pediatric Surgery, the Second Affiliated Hospital of Xi'an Jiaotong University, No. 157, Xiwu Road, Xi'an 710004, Shaanxi, China. ${ }^{2}$ Department of Neonatology, the third Affiliated Hospital, Xi'an Jiaotong University, Xi'an 710068, Shaanxi Province, China. ${ }^{3}$ Department of Respiratory Medicine, the Second Affiliated Hospital of Xi'an Jiaotong University, Xi'an 710004, Shaanxi Province, China.

Received: 20 March 2021 Accepted: 18 November 2021

Published online: 29 November 2021

\section{References}

1. Palla J, Sockrider MM. Congenital lung malformations. Pediatr Ann. 2019;48(4):e169-74.

2. Wong KKY, Flake AW, Tibboel D, Rottier RJ, Tam PKH. Congenital pulmonary airway malformation: advances and controversies. Lancet Child Adolesc Health. 2018:2(4):290-7.

3. Seear M, Townsend J, Hoepker A, Jamieson D, McFadden D, Daigneault $P$, et al. A review of congenital lung malformations with a simplified classification system for clinical and research use. Pediatr Surg Int. 2017;33(6):657-64.

4. Wagner R, Ayoub L, Kahnamoui S, Li H, Patel D, Liu D, et al. Establishment of a biobank for human lung tissues of congenital diaphragmatic hernia and congenital pulmonary airway malformation. J Pediatr Surg. 2019;54(11):2439-42.

5. Taylor B, Rice A, Nicholson AG, Hind M, Dean CH. Mechanism of lung development in the aetiology of adult congenital pulmonary airway malformations. Thorax. 2020;75(11):1001-3.
6. Kapralik J, Wayne C, Chan E, Nasr A. Surgical versus conservative management of congenital pulmonary airway malformation in children: a systematic review and meta-analysis. J Pediatr Surg. 2016;51(3):508-12.

7. Vincent M, Karolak JA, Deutsch G, Gambin T, Popek E, Isidor B, et al. Clinical, Histopathological, and molecular diagnostics in lethal lung developmental disorders. Am J Respir Crit Care Med. 2019;200(9):1093-101.

8. Stocker JT, Madewell JE, Drake RM. Congenital cystic adenomatoid malformation of the lung. Classification and morphologic spectrum. Hum Pathol. 1977:8(2):155-71.

9. Stocker J. Congenital pulmonary airway malformation: A new name for and an expanded classification of congenital cystic adenomatoid malformation of the lung. Sympos 24 Non Neoplastic Lung Dis Histopathol. 2002:41:424-30.

10. Zobel M, Gologorsky R, Lee H, Vu L. Congenital lung lesions. Semin Pediatr Surg. 2019;28(4):150821.

11. Demir OF, Hangul M, Kose M. Congenital lobar emphysema: diagnosis and treatment options. Int J Chron Obstruct Pulmon Dis. 2019;14:921-8.

12. Imai Y, Mark EJ. Cystic adenomatoid change is common to various forms of cystic lung diseases of children: a clinicopathologic analysis of 10 cases with emphasis on tracing the bronchial tree. Arch Pathol Lab Med. 2002;126(8):934-40.

13. Quinn JJ, Chang HY. Unique features of long non-coding RNA biogenesis and function. Nat Rev Genet. 2016;17(1):47-62.

14. Ransohoff JD, Wei Y, Khavari PA. The functions and unique features of long intergenic non-coding RNA. Nat Rev Mol Cell Biol. 2018;19(3):143-57.

15. Beermann J, Piccoli MT, Viereck J, Thum T. Non-coding RNAs in development and disease: background, mechanisms, and therapeutic approaches. Physiol Rev. 2016;96(4):1297-325.

16. Kondo Y, Shinjo K, Katsushima K. Long non-coding RNAs as an epigenetic regulator in human cancers. Cancer Sci. 2017;108(10):1927-33.

17. Qian X, Zhao J, Yeung PY, Zhang QC, Kwok CK. Revealing IncRNA structures and interactions by sequencing-based approaches. Trends Biochem Sci. 2019;44(1):33-52.

18. Mon RA, Johnson KN, Ladino-Torres M, Heider A, Mychaliska GB, Treadwell $M C$, et al. Diagnostic accuracy of imaging studies in congenital lung malformations. Arch Dis Child Fetal Neonatal Ed. 2019;104(4):F372-f377.

19. Joung J, Engreitz JM, Konermann S, Abudayyeh OO, Verdine VK, Aguet F, et al. Genome-scale activation screen identifies a IncRNA locus regulating a gene neighbourhood. Nature. 2017;548(7667):343-6.

20. Zirpoli S, Munari AM, Primolevo A, Scarabello M, Costanzo S, Farolfi A, et al. Agreement between magnetic resonance imaging and computed tomography in the postnatal evaluation of congenital lung malformations: a pilot study. Eur Radiol. 2019;29(9):4544-54.

21. Leblanc C, Baron M, Desselas E, Phan MH, Rybak A, Thouvenin G, et al. Congenital pulmonary airway malformations: state-of-the-art review for pediatrician's use. Eur J Pediatr. 2017:176(12):1559-71.

22. Delacourt C, Bertille N, Salomon LJ, Benachi A, Henry E, Massardier J, et al. Prenatal natural history of congenital pulmonary malformations: MALFPULM population-based cohort study. Ultrasound Obstet Gynecol. 2019;54(3):381-8.

23. Swarr DT, Peranteau WH, Pogoriler J, Frank DB, Adzick NS, Hedrick HL, et al. Novel molecular and phenotypic insights into congenital lung malformations. Am J Respir Crit Care Med. 2018;197(10):1328-39.

24. Cheng J, Chen Y, Zhao P, Liu X, Dong J, Li J, et al. Downregulation of miRNA-638 promotes angiogenesis and growth of hepatocellular carcinoma by targeting VEGF. Oncotarget. 2016;7(21):30702-11.

25. Love Ml, Huber W, Anders S. Moderated estimation of fold change and dispersion for RNA-seq data with DESeq2. Genome Biol. 2014;15(12):550.

26. Kassambara A, Mundt F. factoextra: Extract and Visualize the Results of Multivariate Data Analyses. (R package version 1.0.7); 2020.

27. Murtagh F, Legendre P. Ward's hierarchical agglomerative clustering method: which algorithms implement Ward's criterion? J Classif. 2014;31:274-95.

28. Groemping U. Relative importance for linear regression in $\mathrm{R}$ : the package relaimpo. J Stat Softw. 2006;17(1):925-33.

29. Gu Z, Eils R, Schlesner M. Complex heatmaps reveal patterns and correlations in multidimensional genomic data. Bioinformatics (Oxford, England). 2016:32(18):2847-9.

30. Langfelder P, Horvath S. WGCNA: an R package for weighted correlation network analysis. BMC Bioinformatics. 2008;9:559. 
31. Dirmeier S, Fuchs C, Mueller NS, Theis FJ. netReg: network-regularized linear models for biological association studies. Bioinformatics (Oxford, England). 2018;34(5):896-8.

32. Enright AJ, Van Dongen S, Ouzounis CA. An efficient algorithm for largescale detection of protein families. Nucleic Acids Res. 2002;30(7):1575-84.

33. Kanehisa M, Goto S. KEGG: Kyoto encyclopedia of genes and genomes. Nucleic Acids Res. 2000;28(1):27-30.

34. Zhong $Z$, Hong $M, C$ Chen $X, X i Y, X u Y$, Kong $D$, et al. Transcriptome analysis reveals the link between IncRNA-mRNA co-expression network and tumor immune microenvironment and overall survival in head and neck squamous cell carcinoma. BMC Med Genet. 2020;13(1):57.

35. Wittkopp PJ, Kalay G. Cis-regulatory elements: molecular mechanisms and evolutionary processes underlying divergence. Nat Rev Genet. 2011;13(1):59-69.

\section{Publisher's Note}

Springer Nature remains neutral with regard to jurisdictional claims in published maps and institutional affiliations.

- fast, convenient online submission

- thorough peer review by experienced researchers in your field

- rapid publication on acceptance

- support for research data, including large and complex data types

- gold Open Access which fosters wider collaboration and increased citations

- maximum visibility for your research: over $100 \mathrm{M}$ website views per year

At BMC, research is always in progress.

Learn more biomedcentral.com/submissions 